\title{
TEORES DE NUTRIENTES NAS FOLHAS DE BANANEIRA, CV. PACOVAN, SOB IRRIGAÇÃO
}

\author{
Nutrient levels in banana leaves, cultivar Pacovan, grown under irrigation
}

\author{
Ana Lúcia Borges ${ }^{1}$, Ranulfo Corrêa Caldas ${ }^{2}$
}

\section{RESUMO}

Por meio da análise química dos nutrientes na folha, é possível avaliar o estado nutricional da planta, em que os resultados obtidos são comparados a padrões que se baseiam na correlação entre a concentração do nutriente nas folhas e o desenvolvimento ou produção da cultura. Objetivou-se definir os teores padrões de macro e micronutrientes nas folhas de bananeiras cv. Pacovan sob irrigação, no pólo Petrolina-PE/Juazeiro-BA. Nos bananais selecionados, foram marcadas 20 bananeiras e amostradas as folhas na época do florescimento, para análise química, e anotado o peso dos cachos de dois ciclos de produção subseqüentes. Realizou-se a análise estatística descritiva, com os dados de plantas com pesos de cachos em quatro estratos $(\leq 10 ;>10$ e $\leq 15 ;>15$ e $\leq 20 ;>20 \mathrm{~kg}$ ), considerando os cachos superiores a $20 \mathrm{~kg}$ como em condições ideais de nutrição. As faixas dos teores padrões definidas para cv. Pacovan, sob irrigação, no Distrito de Irrigação Senador Nilo Coelho, em Petrolina-PE, foram: 22-24 g de N/kg; 1,7-1,9 g de P/kg; 25-28 g de K/kg; 6,3-7,3 g de Ca/kg; 3,13,5 g de Mg/kg; 1,7-1,9 g de S/kg; 13-16 mg de B/kg; 6-7 mg de Cu/kg; 71-86 mg de Fe/kg; 315-398 mg de Mn/kg; e 12$14 \mathrm{mg}$ de $\mathrm{Zn} / \mathrm{kg}$.

Termos para indexação: Musa sp., teor padrão, macronutriente, micronutriente, nutrição.

\section{ABSTRACT}

Chemical analyses of nutrient contents in leaves is possible to indicate the plant nutritional state, where the results is compared with a standard. For results interpretation it is necessary the establishment standard values that are based on correlation studies between nutrient concentration in the leaves and crop development or yield. The objective of this work was to establish critical levels of macro and micronutrients in banana leaves, cultivar Pacovan, grown under irrigation in the PetrolinaPE/Juazeiro-BA region. In selected banana orchards 20 plants were chosen for further evaluations. Leaf sample were collected at flowering for chemical analyses and bunch weight determined. Evaluations were carried out during two production cycles. Descriptive statistical analysis was performed considering four extracts of bunch weight $(\leq 10 ;>10$ and $\leq 15 ;>15$ and $\leq 20$; > $20 \mathrm{~kg}$ ), and plants that produced bunch $20 \mathrm{~kg}$ heavier express an ideal nutritional conditions. The nutrient levels determined for the banana cultivar Pacovan, grown under irrigation, in the Petrolina-Juazeiro region, were the following: $22-24 \mathrm{~g}$ of N/kg; $1,7-$ $1,9 \mathrm{~g}$ of P/kg; $25-28 \mathrm{~g}$ of $\mathrm{K} / \mathrm{kg} ; 6,3-7,3 \mathrm{~g}$ of $\mathrm{Ca} / \mathrm{kg} ; 3,1-3,5 \mathrm{~g}$ of $\mathrm{Mg} / \mathrm{kg} ; 1,7-1,9 \mathrm{~g}$ of S/kg; $13-16 \mathrm{mg}$ of B/kg; $6-7 \mathrm{mg}$ of Cu/kg; $71-86 \mathrm{mg}$ of $\mathrm{Fe} / \mathrm{kg} ; 315-398 \mathrm{mg}$ of $\mathrm{Mn} / \mathrm{kg}$; and $12-14 \mathrm{mg}$ of $\mathrm{Zn} / \mathrm{kg}$.

Index terms: Musa sp., standard level, macronutrient, micronutrient, nutrition.

(Recebido para publicação em 28 de julho de 2003 e aprovado em 26 de agosto de 2004)

\section{INTRODUÇÃO}

A fruticultura irrigada encontra-se em franca expansão nas regiões semi-áridas do Nordeste brasileiro, principalmente nos pólos de Petrolina-Juazeiro (PEBA), Jaíba (MG) e Vale do Açu (RN), em razão de suas características climáticas. A Região Nordeste representa $34,3 \%$ da produção nacional de banana (IBGE, 2002) e grande parte sob condições de irrigação.

A diagnose foliar tem sido bastante útil na avaliação quantitativa do estado nutricional das culturas e nas recomendações de adubação, sendo o teor do nutriente na planta resultante da ação e interação dos fatores que afetam a sua disponibilidade no solo e absorção pela planta (MUNSON e NELSON, 1973).

Por meio da análise química dos nutrientes na folha, órgão que apresenta maior atividade fisiológica, avalia-se o estado nutricional da planta, comparando-se com um padrão. Para interpretação dos resultados obtidos, estabelecem-se teores padrões ou faixas de referência, comparando-os com os padrões já existentes, baseando-se na correlação entre a concentração do nutriente nas folhas e o desenvolvimento ou a produção da cultura. Os teores padrões de referência têm aplicação universal, embora estejam sempre relacionados

\footnotetext{
1. Engenheira Agrônoma, Pesquisadora da Embrapa Mandioca e Fruticultura, Caixa Postal 007 - 44380-000 - Cruz das Almas, BA. analucia@ cnpmf.embrapa.br.

2. Engenheiro Agrônomo, Pesquisador da Embrapa Mandioca e Fruticultura, rcaldas@cnpmf.embrapa.br.
} 
com fatores ambientais, solo, cultivares etc. Assim, os valores de referência quase sempre precisam ser adaptados às condições locais, embora alguma extrapolação sempre seja possível (BATAGLIA e DECHEN, 1986). Dessa maneira, é importante estabelecer, para diferentes cultivares de banana, a concentração do nutriente abaixo do qual espera-se resposta à aplicação de adubo.

Quanto aos teores padrões, Lahav e Turner (1983) apresentam os valores médios obtidos por vários autores, na terceira folha de bananeiras do subgrupo Cavendish (grupo AAA), para os macronutrientes, em $\mathrm{g} / \mathrm{kg}$ : 26,0 de nitrogênio $(\mathrm{N}) ; 2,0$ de fósforo $(\mathrm{P}) ; 30,0 \mathrm{de}$ potássio $(\mathrm{K})$; 5,0 de cálcio $(\mathrm{Ca})$; 3,0 de magnésio $(\mathrm{Mg})$ e 2,3 de enxofre (S); e para os micronutrientes, em $\mathrm{mg} / \mathrm{kg}$ : 11 de boro (B); 9 de cobre $(\mathrm{Cu}) ; 80$ de ferro $(\mathrm{Fe}) ; 25$ de manganês $(\mathrm{Mn})$ e 18 de zinco $(\mathrm{Zn})$, empregados para interpretação dos resultados da análise foliar da bananeira.

Além desses valores, IFA (1992) indica os teores ótimos de nutrientes padronizados para a parte interna do limbo da terceira folha, no estádio da inflorescência descoberta (amostra internacional de referência), para os macronutrientes, em $\mathrm{g} / \mathrm{kg}$ : 27,036,0 de N; 1,6-2,7 de P; 32,0-54,0 de K; 6,6-12,0 de $\mathrm{Ca} ; 2,7-6,0$ de $\mathrm{Mg}$ e $1,6-3,0 \mathrm{~g}$ de $\mathrm{S}$; e para os micronutrientes, em mg/kg: $10-25$ de B; 6-30 de $\mathrm{Cu} ; 80$ 360 de Fe; 200-1.800 de Mn e 20-50 de Zn, os quais têm sido também utilizados nas interpretações dos resultados.

Para as condições brasileiras, Teixeira et al. (2002), objetivando avaliar o desempenho de diagnósticos nutricionais em bananeira 'Nanicão' estabelecidos a partir do critério de níveis críticos de $\mathrm{N}$ e $\mathrm{K}$ e do sistema integrado de diagnose e recomendação (DRIS), constataram que a eficiência dos diagnósticos com DRIS para $\mathrm{N}$ foi superior à daqueles baseados no critério de nível crítico (NC), e para K, tanto o DRIS como o critério de $\mathrm{NC}$ apresentaram desempenhos semelhantes. O potássio é o nutriente mais absorvido pela bananeira; apesar de não fazer parte de compostos na planta, apresenta funções importantes como ativador enzimático, em processos como abertura e fechamento dos estômatos, fotossíntese e transporte de carboidratos. É um nutriente importante na translocação de fotossintatos, no balanço de água, na produção de cachos e pencas, na qualidade e resistência dos frutos, acelerando também o seu desenvolvimento e maturação (BORGES et al., 1995).

Em levantamento realizado nos bananais 'Prata Anã' irrigados do norte de Minas Gerais, Silva e Rodrigues (2001) observaram deficiência de nutrientes, principalmente micronutrientes, e dentre esses, o Zn (18,7-
$19,7 \mathrm{mg} / \mathrm{kg}$ ), nas amostras foliares. Entre os macronutrientes, o cálcio apresentou-se adequado em $98 \%$ das amostras foliares, com teores de 5,9-6,1 g/kg. O Ca tem função estrutural na planta, sendo constituinte dos pectatos de Ca da lamela média das células (BORGES et al., 1995).

Silva et al. (2002) estabeleceram para bananais 'Prata Anã' cultivados no norte de Minas Gerais as seguintes faixas de suficiência para os macronutrientes, em $\mathrm{g} / \mathrm{kg}$ : $\mathrm{N}=25,0-29,0 ; \mathrm{P}=1,5-1,9 ; \mathrm{K}=27,0-35,0$; $\mathrm{Ca}=4,5-7,5 ; \mathrm{Mg}=2,4-4,0$ e $\mathrm{S}=1,7-2,0$, e para os micronutrientes, em $\mathrm{mg} / \mathrm{kg}: \mathrm{B}=25-32 ; \mathrm{Cu}=2,6-8,8$; $\mathrm{Fe}=72-157 ; \mathrm{Na}=20-60 ; \mathrm{Mn}=173-630$ e $\mathrm{Zn}=14-25$.

No Estado do Rio de Janeiro, Fontes et al. (2003), avaliando o estado nutricional e o efeito da aplicação de $\mathrm{N}$ no desenvolvimento da bananeira 'Prata Anã $\tilde{}^{\prime}$, verificaram que as doses de $\mathrm{N}$ aumentaram o teor de Mn e reduziu o de cloro. O Mn é um ativador enzimático, participando de reações bioquímicas da fotossíntese e respiração. A deficiência dele no solo ocorre em condições de calagem excessiva e muita matéria orgânica.

Objetivou-se neste trabalho definir teores padrões de macro e micronutrientes nas folhas de bananeira cv. Pacovan irrigada, no pólo Petrolina-PE/JuazeiroBA.

\section{MATERIAL E MÉTODOS}

No período de maio a julho/1999, realizaram-se o levantamento dos municípios produtores de banana e a seleção das áreas representativas do pólo de irrigação Petrolina-PE/Juzeiro-BA. Selecionou-se como área representativa da região o Distrito de Irrigação Senador Nilo Coelho, com 2.800 ha de banana 'Pacovan', sendo $90 \%$ de pequenos produtores. O Distrito conta com onze núcleos, sendo selecionados dez desses. O número de lotes amostrados em cada núcleo foi estabelecido com base na variável quantidade de bananeiras existente. No período de agosto/1999 a março/2000, foram aplicados os questionários com a finalidade de obterem-se informações do histórico das áreas (espaçamento, sistema de irrigação, práticas culturais, manejo de pragas e doenças, uso de calcário, de fertilizante e dados de produção).

Os bananais foram selecionados ao acaso em cada núcleo, no total de 185 . Em cada bananal selecionado, foram marcadas 20 bananeiras e amostradas as folhas na época do florescimento (todas as pencas femininas descobertas e não mais de duas masculinas), segundo metodologia descrita em Borges et al. (1995), para as análises químicas dos macronutrientes (N, P, K, Ca, Mg e S) e dos micronutrientes (B,

Ciênc. agrotec., Lavras, v. 28, n. 5, p. 1099-1106, set.out., 2004 
$\mathrm{Cu}, \mathrm{Fe}, \mathrm{Mn}$ e $\mathrm{Zn}$ ), realizadas segundo Bataglia et al. (1983). Os cachos das plantas selecionadas, de dois ciclos subseqüentes à marcação das plantas, foram colhidos, despencados, pesados e classificados em quatro estratos: < $10 \mathrm{~kg}$; > $10 \mathrm{e} \leq 15 \mathrm{~kg} ; 15 \mathrm{e} \leq$ $20 \mathrm{~kg}$ e $>20 \mathrm{~kg}$. Amostras de solo foram retiradas das áreas e as propriedades químicas estão apresentadas na Tabela 1, de acordo com o peso dos cachos.

Realizou-se a análise estatística descritiva dos dados das plantas com pesos de cachos nos quatro estratos: $\leq 10$ (22 plantas); > 10 e $\leq 15$ (72 plantas); > 15 e $\leq 20$ (62 plantas); > 20 (29 plantas), considerando cachos superiores a $20 \mathrm{~kg}$ como em condições ideais de nutrição. Foram estimados os coeficientes de correlação linear simples (Pearson) entre os macro e micronutrientes da terceira folha da bananeira 'Pacovan' com cachos $>20 \mathrm{~kg}$.

\section{RESULTADOS E DISCUSSÃO}

Dos 185 bananais amostrados, 29 apresentaram pesos de cachos iguais ou superiores a $20 \mathrm{~kg}$, correspondendo a $15,7 \%$ das áreas (Figura 1). O peso médio dos cachos nesse estrato foi de $22,7 \mathrm{~kg}$, o mínimo de $20,1 \mathrm{~kg}$ e o máximo de $30,1 \mathrm{~kg}$, com o coeficiente de variação de $11,3 \%$ e intervalo de confiança de 0,97 a $5 \%$ de probabilidade.

Os teores médios de macronutrientes encontrados nas folhas de bananeira cv. Pacovan sob irrigação, em plantas com peso de cacho inferior e igual a $20 \mathrm{~kg}$, estão apresentados na Tabela 2, e naqueles com cachos superiores a $20 \mathrm{~kg}$, na Tabela 3. Exceto para Ca, o CV foi menor nas plantas com cachos maiores que $20 \mathrm{~kg}$. $\mathrm{Na}$ maioria dos casos, foram observados teores de macronutrientes mais elevados nas plantas com cachos menores (Tabela 2), em comparação com aquelas

TABELA 1 - Propriedades químicas do solo, das áreas amostradas, na camada de 0-20 cm de profundidade, nos quatro estratos, em solos do Distrito de Irrigação Senador Nilo Coelho, em Petrolina-PE. 1999-2000.

Solo com cachos $\leq 10 \mathrm{~kg}$ :

\begin{tabular}{|c|c|c|c|c|c|c|c|c|c|}
\hline pH água & P (Mehlich) & $\mathbf{K}$ & $\mathbf{C a}$ & Mg & Al & $\mathbf{S}$ & CTC & $\mathbf{V}$ & $\mathbf{M O}^{1}$ \\
\hline & $\mathrm{mg} / \mathrm{dm}^{3}$ & \multicolumn{6}{|c|}{--------------------- $\mathrm{cmol}_{\mathrm{c}} / \mathrm{dm}^{3}$------------------- } & $\%$ & $-\mathrm{g} / \mathrm{kg}-$ \\
\hline 5,4 & 36 & 106 & 1,70 & 0,47 & 0,06 & 2,53 & 3,79 & 66 & 6,35 \\
\hline \multicolumn{10}{|c|}{ Solo com cachos $>10$ e $\leq 15 \mathrm{~kg}$ : } \\
\hline \multirow[t]{2}{*}{ pH água } & P (Mehlich) & $\mathbf{K}$ & $\mathbf{C a}$ & Mg & Al & $\mathbf{S}$ & CTC & $\mathbf{V}$ & $\mathbf{M O}^{1}$ \\
\hline & $\mathrm{mg} / \mathrm{dm}^{3}$ & \multicolumn{6}{|c|}{ 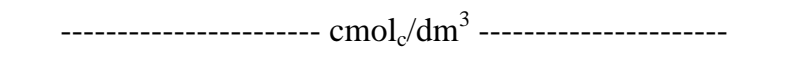 } & $\%$ & $-\mathrm{g} / \mathrm{kg}-$ \\
\hline 5,3 & 46 & 126 & 2,06 & 0,63 & 0,09 & 3,08 & 4,60 & 66 & 7,50 \\
\hline \multicolumn{10}{|c|}{ Solo com cachos $>15$ e $\leq 20 \mathrm{~kg}$ : } \\
\hline \multirow[t]{2}{*}{ pH água } & P (Mehlich) & $\mathbf{K}$ & $\mathbf{C a}$ & Mg & Al & $\mathbf{S}$ & CTC & $\mathbf{V}$ & $\mathbf{M O}^{1}$ \\
\hline & $\mathrm{mg} / \mathrm{dm}^{3}$ & \multicolumn{6}{|c|}{ 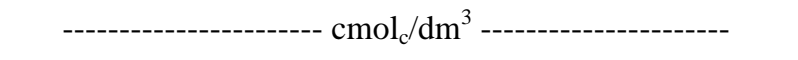 } & $\%$ & $-\mathrm{g} / \mathrm{kg}-$ \\
\hline 5,6 & 41 & 115 & 2,17 & 0,68 & 0,06 & 3,20 & 4,59 & 70 & 7,20 \\
\hline \multicolumn{10}{|c|}{ Solo com cachos > $20 \mathrm{~kg}$ : } \\
\hline \multirow[t]{2}{*}{ pH água } & P (Mehlich) & $\mathbf{K}$ & $\mathbf{C a}$ & Mg & Al & $\mathbf{S}$ & CTC & $\mathbf{V}$ & $\mathbf{M O}^{1}$ \\
\hline & $\mathrm{mg} / \mathrm{dm}^{3}$ & \multicolumn{6}{|c|}{ 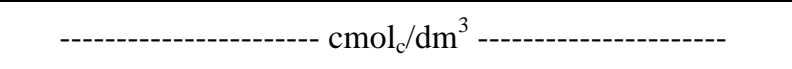 } & $\%$ & $-\mathrm{g} / \mathrm{kg}-$ \\
\hline 5,6 & 44 & 156 & 2,30 & 0,77 & 0,05 & 3,54 & 4,87 & 72 & 7,63 \\
\hline
\end{tabular}

${ }^{1}$ Matéria orgânica. 
com cachos maiores (Tabela 3), indicando o efeito concentração, ou seja, o menor desenvolvimento da planta levou a uma maior concentração do nutriente nela.

Os teores mínimo e médio de nitrogênio $(\mathrm{N})$ nas folhas das plantas com cachos $>20 \mathrm{~kg}$ (Tabela 3 ) estiveram abaixo daqueles estabelecidos por Lahav e Turner (1983), IFA (1992) e Silva et al. (2002). No entanto, o teor máximo $(27,4 \mathrm{~g} / \mathrm{kg})$ encontrou-se na faixa proposta por esses autores. $\mathrm{O} \mathrm{N}$ correlacionouse positivamente com o cálcio $(\mathrm{Ca})$ e negativamente com o boro (B), ou seja, quanto maior o teor de $\mathrm{N}$ nas folhas, maior a concentração de $\mathrm{Ca}$ e menor a de B (Tabela 4).

Os teores mínimo e médio de fósforo $(\mathrm{P})$ encontrados (Tabela 3) estiveram abaixo do estabelecido por Lahav e Turner (1983) para bananeiras do subgrupo Cavendish, mas o valor médio encontrou-se na faixa indicada por IFA (1992) e Silva et al. (2002). Os teores máximos de $\mathrm{P}$ obtidos foram superiores nas plantas com cachos menores, apesar de os teores no solo estarem adequados, ou seja, acima de $30 \mathrm{mg} / \mathrm{dm}^{3}$ (Tabela 1). O P correlacionou-se positivamente com o magnésio $(\mathrm{Mg})$ e o boro e negativamente com o potássio (K) (Tabela 4).

Os teores de $\mathrm{K}$ foram superiores nas plantas com cachos maiores que $20 \mathrm{~kg}$ (Tabela 3), como também os teores no solo (Tabela 1).

Exceto o teor máximo de $\mathrm{K}$ encontrado nas folhas, os teores mínimo e médio estiveram abaixo dos estabelecidos por Lahav e Turner (1983), IFA (1992) e Silva et al. (2002). Observou-se correlação linear positiva e significativa com o $\mathrm{Ca}, \mathrm{B}$ e ferro ( $\mathrm{Fe}$ ), e negativa com o P (Tabela 4). Os teores de $\mathrm{Ca}$ e $\mathrm{Mg}$ nos solos dos bananais com cachos superiores a 20 $\mathrm{kg}$ foram mais elevados (Tabela 1), sendo importante salientar que, em solos com calagem excessiva, portanto com altos teores de $\mathrm{Ca}$ e $\mathrm{Mg}$, pode ocorrer deficiência de K (MALAVOLTA et al., 1989).

Os teores de $\mathrm{Ca}$ encontrados nas folhas (Tabela 3) estiveram dentro das faixas estabelecidas por Lahav e Turner (1983) e IFA (1992), exceto o valor mínimo, e acima dos teores estabelecidos para 'Prata Anã' por Silva et al. (2002). Obteve-se correlação linear simples significativa e positiva com o $\mathrm{N}, \mathrm{K}$ e $\mathrm{B}$, e negativa com o Mg (Tabela 4). Sabe-se a importância do balanço das bases (K, Ca e $\mathrm{Mg})$ na bananeira.

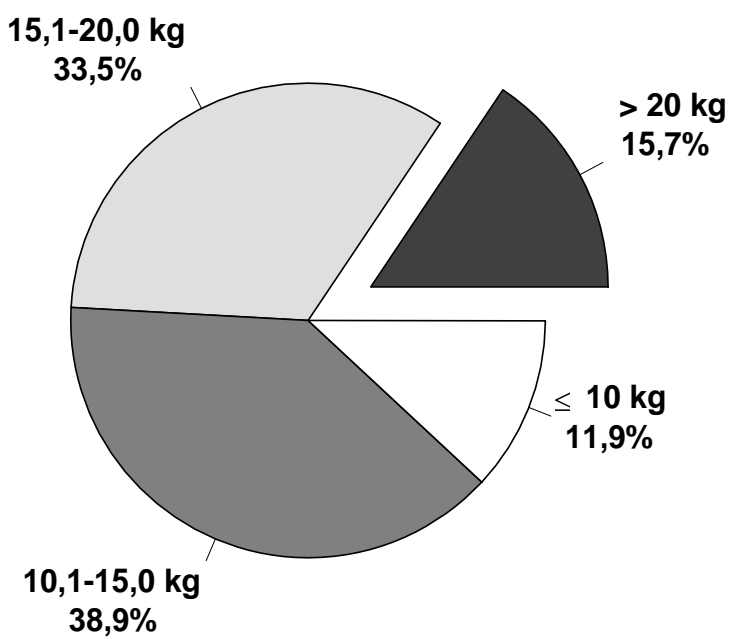

FIGURA 1 - Distribuição percentual dos cachos $(\mathrm{kg})$ nos 185 bananais amostrados. Média de dois ciclos de produção.

Ciênc. agrotec., Lavras, v. 28, n. 5, p. 1099-1106, set.out., 2004 
TABELA 2 - Teores de macronutrientes nas folhas de bananeiras cv. Pacovan sob irrigação, em três estratos de pesos de cachos, no Distrito de Irrigação Senador Nilo Coelho, em Petrolina - PE. Média de dois ciclos de produção.

Cachos com pesos $\leq 10 \mathrm{~kg}$ :

\begin{tabular}{|c|c|c|c|c|c|c|}
\hline & \multicolumn{6}{|c|}{ Macronutrientes } \\
\hline & $\mathbf{N}$ & $\mathbf{P}$ & $\mathbf{K}$ & $\mathbf{C a}$ & Mg & $\mathbf{S}$ \\
\hline \multicolumn{7}{|c|}{ - } \\
\hline Teor médio & 22,3 & 1,9 & 23,5 & 6,9 & 3,1 & 2,0 \\
\hline Teste $\mathrm{t}^{1}$ & $* *$ & $* *$ & $* *$ & $* *$ & $* *$ & $* *$ \\
\hline $\mathrm{CV}(\%)$ & 14,2 & 18,7 & 12,8 & 16,7 & 18,6 & 12,2 \\
\hline \multicolumn{7}{|c|}{ Cachos com pesos $>10$ e $\leq 15 \mathrm{~kg}$ : } \\
\hline & \multicolumn{6}{|c|}{ Macronutrientes } \\
\hline & $\mathbf{N}$ & $\mathbf{P}$ & $\mathbf{K}$ & $\mathbf{C a}$ & Mg & $\mathbf{S}$ \\
\hline \multicolumn{7}{|c|}{ - } \\
\hline Teor médio & 22,6 & 1,9 & 24,6 & 7,0 & 3,4 & 1,9 \\
\hline Teste $\mathrm{t}^{1}$ & $* *$ & $* *$ & $* *$ & $* *$ & $* *$ & $* *$ \\
\hline $\mathrm{CV}(\%)$ & 14,2 & 16,7 & 10,7 & 17,6 & 13,2 & 11,1 \\
\hline \multicolumn{7}{|c|}{ Cachos com pesos $>15 \mathrm{e} \leq 20 \mathrm{~kg}$ : } \\
\hline & \multicolumn{6}{|c|}{ Macronutrientes } \\
\hline & $\mathbf{N}$ & $\mathbf{P}$ & $\mathbf{K}$ & $\mathbf{C a}$ & Mg & $\mathbf{S}$ \\
\hline \multicolumn{7}{|c|}{ 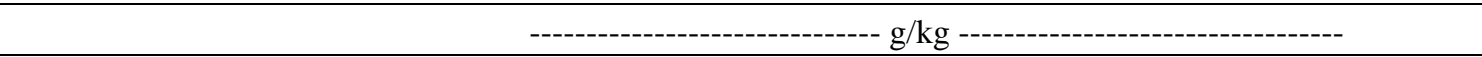 } \\
\hline Teor médio & 21,9 & 1,8 & 24,1 & 7,1 & 3,4 & 1,8 \\
\hline Teste $\mathrm{t}^{1}$ & $* *$ & $* *$ & $* *$ & $* *$ & $* *$ & $* *$ \\
\hline $\mathrm{CV}(\%)$ & 12,0 & 15,2 & 10,9 & 17,9 & 12,8 & 10,7 \\
\hline
\end{tabular}

$\mathbf{1}_{* * *}(\alpha \leq \mathbf{0 , 0 1})$

TABELA 3 - Teores de macronutrientes nas folhas de bananeiras cv. Pacovan sob irrigação, com pesos de cachos superiores a 20 kg, no Distrito de Irrigação Senador Nilo Coelho, em Petrolina - PE. Média de dois ciclos de produção.

\begin{tabular}{|c|c|c|c|c|c|c|}
\hline & \multicolumn{6}{|c|}{ Macronutrientes } \\
\hline & $\mathbf{N}$ & $\mathbf{P}$ & $\mathbf{K}$ & $\mathbf{C a}$ & Mg & $\mathbf{S}$ \\
\hline & \multicolumn{6}{|c|}{ 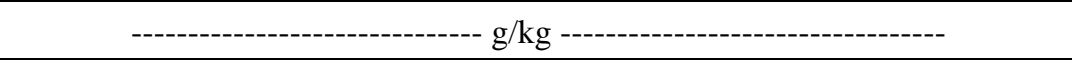 } \\
\hline Teor médio & 23,1 & 1,8 & 26,5 & 6,8 & 3,3 & 1,8 \\
\hline Teor máximo & 27,4 & 2,5 & 32,6 & 10,3 & 4,0 & 2,5 \\
\hline Teor mínimo & 17,7 & 1,5 & 21,8 & 4,6 & 2,3 & 1,5 \\
\hline Teste $\mathrm{t}^{1}$ & $* *$ & $* *$ & $* *$ & $* *$ & $* *$ & $* *$ \\
\hline $\begin{array}{l}\text { Intervalo de Confiança } \\
(\alpha \leq 0,05)\end{array}$ & 0,92 & 0,07 & 1,06 & 0,54 & 0,17 & 0,07 \\
\hline $\mathrm{CV}(\%)$ & 10,5 & 10,9 & 10,5 & 20,7 & 13,7 & 10,0 \\
\hline
\end{tabular}

\footnotetext{
$1 * *(\alpha \leq \mathbf{0 , 0 1})$
} 
TABELA 4 - Estimativas dos coeficientes de correlação (r) linear simples entre os teores de macro e micronutrientes na terceira folha da bananeira cv. Pacovan com cachos $>20 \mathrm{~kg}$.

\begin{tabular}{|c|c|c|c|c|c|c|c|c|c|c|c|}
\hline & $\mathbf{N}$ & $\mathbf{P}$ & $\mathbf{K}$ & $\mathrm{Ca}$ & Mg & $\mathbf{S}$ & B & $\mathrm{Cu}$ & $\mathrm{Fe}$ & Mn & $\mathbf{Z n}$ \\
\hline $\mathrm{N}$ & - & NS & NS & $0,25^{* *}$ & NS & NS & $-0,22 * *$ & NS & NS & NS & NS \\
\hline $\mathrm{P}$ & & - & $-0,19 * *$ & NS & $0,21 * *$ & NS & $0,27 * *$ & NS & NS & NS & NS \\
\hline $\mathrm{K}$ & & & - & $0,27 * *$ & NS & NS & $0,44 * *$ & NS & $0,34 * *$ & NS & NS \\
\hline $\mathrm{Ca}$ & & & & - & $-0,46^{* *}$ & NS & $0,24 * *$ & NS & NS & NS & NS \\
\hline $\mathrm{Mg}$ & & & & & - & $0,45^{* *}$ & NS & NS & NS & $0,24 * *$ & NS \\
\hline$S$ & & & & & & - & NS & $0,19 * *$ & NS & NS & NS \\
\hline B & & & & & & & - & NS & NS & $0,21 * *$ & NS \\
\hline $\mathrm{Cu}$ & & & & & & & & - & NS & NS & NS \\
\hline $\mathrm{Fe}$ & & & & & & & & & - & NS & $0,29 * *$ \\
\hline $\mathrm{Mn}$ & & & & & & & & & & - & NS \\
\hline
\end{tabular}

O teor médio de Mg obtido para a cv. Pacovan (Tabela 3) apresentou-se dentro das faixas estabelecidas por Lahav e Turner (1983), IFA (1992) e Silva et al. (2002). O teor mínimo de $\mathrm{Mg}$ nas folhas nas plantas com cachos entre $>15 \mathrm{e} \leq 20 \mathrm{~kg}$ foi superior ao das plantas com cachos > $20 \mathrm{~kg}$, apesar de o teor de $\mathrm{Mg}$ nesses solos ser mais elevado do que nos demais. $\mathrm{O} \mathrm{Mg}$ apresentou correlação linear simples positiva com o $\mathrm{P}$, enxofre (S) e manganês (Mn), e negativa com o Ca (Tabela 4).

O teor médio de enxofre (S) encontrado (Tabela 3) esteve dentro da faixa estabelecida por IFA (1992) e Silva et al. (2002), mas foi $22 \%$ inferior ao valor estabelecido por Lahav e Turner (1983). Observou-se correlação linear simples significativa e positiva do $\mathrm{S}$ com o $\mathrm{Mg}$ e o cobre $(\mathrm{Cu})$ (Tabela 4$)$.

Quanto aos micronutrientes (Tabelas 5 e 6), em geral, nos plantios de banana, as deficiências mais comuns são de boro (B) e zinco (Zn). Os teores de B encontrados estiveram dentro das faixas estabelecidas por Lahav e Turner (1983) e IFA (1992) e inferiores aos valores obtidos por Silva et al. (2002). Entretanto, o teor máximo de $\mathrm{B}$ encontrado está acima do maior valor estabelecido por IFA (1992). O B correlacionou positivamente com $\mathrm{P}, \mathrm{K}, \mathrm{Ca}$ e $\mathrm{Mn}$, e negativamente com o N.

Os teores de $\mathrm{Zn}$ encontrados nas folhas de bananeira 'Pacovan' estiveram abaixo das faixas propostas por Lahav e Turner (1983), IFA (1992) e Silva et al. (2002), exceto o valor máximo $(18 \mathrm{mg} / \mathrm{kg})$, o qual correspondeu ao valor médio proposto por Lahav e Turner (1983) e enquadrou-se na faixa proposta por Silva et al.
(2002). Observou-se correlação positiva do Zn apenas com o ferro. No entanto, a presença desse nutriente no solo é influenciada pelo $\mathrm{pH}$, teores de $\mathrm{P}$, argila e matéria orgânica, cujos valores elevados inibem a absorção do Zn (MALAVOLTA et al., 1989); possivelmente, por isso, os valores encontrados nas plantas com cachos $>20$ $\mathrm{kg}$ foram menores em razão de o $\mathrm{pH}$ do solo ser mais elevado, bem como os teores de $\mathrm{P}$ e matéria orgânica (Tabela 1). Os valores são inferiores aos obtidos por Silva e Rodrigues (2001) no norte de Minas Gerais para 'Prata Anã'.

O valor mínimo de cobre $(4 \mathrm{mg} / \mathrm{kg})$ encontrado situou-se abaixo da faixa estabelecida por IFA (1992), apesar de essa ser bastante ampla. Esse nutriente correlacionou-se apenas com $\mathrm{S}$.

Os teores médio $(78,2 \mathrm{mg} / \mathrm{kg})$ e mínimo $(50,0$ $\mathrm{mg} / \mathrm{kg}$ ) de ferro encontrados nas folhas situaram-se abaixo dos valores propostos por Lahav e Turner (1983) e IFA (1992). O valor máximo esteve dentro da faixa estabelecida por Silva et al. (2002). O Fe correlacionou-se positivamente com o $\mathrm{K}$ e o $\mathrm{Zn}$.

O valor médio e a faixa para manganês apresentados por Lahav e Turner (1983), IFA (1992) e Silva et al. (2002) são bastante distintos e muito amplos, no caso desses últimos autores. Assim, os valores encontrados na cv. Pacovan estiveram bem acima do proposto por Lahav e Turner (1983); contudo, exceto o valor mínimo, os demais encontraram-se dentro das faixas estabelecidas por IFA (1992) e Silva et al. (2002). 
TABELA 5 - Teores de micronutrientes nas folhas de bananeiras cv. Pacovan no Distrito de Irrigação Senador Nilo Coelho, em Petrolina - PE. Média de dois ciclos de produção.

Cachos com pesos $\leq 10 \mathrm{~kg}$ :

\begin{tabular}{|c|c|c|c|c|c|}
\hline & \multicolumn{5}{|c|}{ Micronutrientes } \\
\hline & B & $\mathbf{C u}$ & Fe & Mn & $\mathbf{Z n}$ \\
\hline \multicolumn{6}{|c|}{------------------------------- mg/kg ----------------------------- } \\
\hline Teor médio & 14,1 & 5,7 & 78,7 & 309,7 & 14,0 \\
\hline Teste $\mathrm{t}^{1}$ & $* *$ & $* *$ & $* *$ & $* *$ & $* *$ \\
\hline $\mathrm{CV}(\%)$ & 13,7 & 19,9 & 21,8 & 43,1 & 28,5 \\
\hline \multicolumn{6}{|c|}{ Cachos com pesos $>10$ e $\leq 15 \mathrm{~kg}$ : } \\
\hline & \multicolumn{5}{|c|}{ Micronutrientes } \\
\hline & B & $\mathbf{C u}$ & $\mathbf{F e}$ & Mn & $\mathbf{Z n}$ \\
\hline \multicolumn{6}{|c|}{ 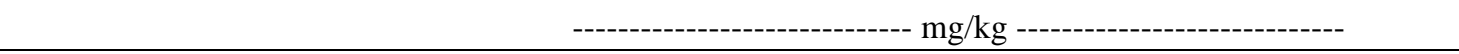 } \\
\hline Teor médio & 13,5 & 6,0 & 78,7 & 373,6 & 14,1 \\
\hline Teste $\mathrm{t}^{1}$ & $* *$ & $* *$ & $* *$ & $* *$ & $* *$ \\
\hline $\mathrm{CV}(\%)$ & 12,1 & 16,7 & 27,4 & 32,0 & 25,8 \\
\hline \multicolumn{6}{|c|}{ Cachos com pesos > 15 e $\leq 20 \mathrm{~kg}$ : } \\
\hline & \multicolumn{5}{|c|}{ Micronutrientes } \\
\hline & B & $\mathbf{C u}$ & $\mathbf{F e}$ & Mn & $\mathbf{Z n}$ \\
\hline \multicolumn{6}{|c|}{ - - } \\
\hline Teor médio & 13,2 & 6,1 & 80,5 & 360,7 & 13,7 \\
\hline Teste $\mathrm{t}^{1}$ & $* *$ & $* *$ & $* *$ & $* *$ & $* *$ \\
\hline $\mathrm{CV}(\%)$ & 12,2 & 21,1 & 30,7 & 29,8 & 22,7 \\
\hline
\end{tabular}

$1 * *(\alpha \leq \mathbf{0 , 0 1})$

TABELA 6 - Teores de micronutrientes nas folhas de bananeiras cv. Pacovan sob irrigação no Distrito de Irrigação Senador Nilo Coelho, em Petrolina - PE. Média de dois ciclos de produção.

\begin{tabular}{lccccc}
\hline & \multicolumn{5}{c}{ Micronutrientes } \\
\cline { 2 - 6 } & $\mathbf{B}$ & $\mathbf{C u}$ & $\mathbf{F e}$ & $\mathbf{M n}$ & $\mathbf{Z n}$ \\
\hline Teor médio & 14,7 & 6,1 & 78,2 & 356,4 & 13,3 \\
Teor máximo & 29,0 & 8,0 & 137,0 & 531,0 & 18,0 \\
Teor mínimo & 11,0 & 4,0 & 50,0 & 139,0 & 9,0 \\
Teste t & & $* *$ & $* *$ & $* *$ & $* *$ \\
Intervalo de Confiança $(\alpha \leq 0,05)$ & 1,53 & 0,41 & 7,54 & 41,49 & 1,18 \\
CV $(\%)$ & 27,3 & 17,6 & 25,3 & 30,6 & 23,3 \\
\hline
\end{tabular}

$1_{* *}(\alpha \leq \mathbf{0 , 0 1})$ 


\section{CONCLUSÃO}

Para as condições do pólo de irrigação PetrolinaPE/Juazeiro-BA, em que foi realizado o trabalho, os teores padrões de nutrientes definidos para cv. Pacovan foram: 22-24 g de N/kg; 1,7-1,9 g de P/kg; 25-28 g de $\mathrm{K} / \mathrm{kg}$; 6,3-7,3 g de $\mathrm{Ca} / \mathrm{kg} ; 3,1-3,5 \mathrm{~g}$ de $\mathrm{Mg} / \mathrm{kg} ; 1,7-1,9 \mathrm{~g}$ de $\mathrm{S} / \mathrm{kg} ; 13-16 \mathrm{mg}$ de $\mathrm{B} / \mathrm{kg} ; 6-7 \mathrm{mg}$ de $\mathrm{Cu} / \mathrm{kg} ; 71-86 \mathrm{mg}$ de $\mathrm{Fe} / \mathrm{kg} ; 315-398 \mathrm{mg}$ de $\mathrm{Mn} / \mathrm{kg}$; e $12-14 \mathrm{mg}$ de $\mathrm{Zn} / \mathrm{kg}$.

\section{REFERÊNCIAS BIBLIOGRÁFICAS}

BATAGLIA, O. C.; DECHEN, A. R. Critérios alternativos para diagnose foliar. In: SIMPÓSIO AVANÇADO DE QUÍMICA E FERTILIDADE DO SOLO, 1., 1986, Piracicaba, SP. Anais... Piracicaba: Fundação Cargill, 1986. p. 115-136.

BATAGLIA, O. C.; FURLANI, A. M.; TEIXEIRA, J. P. F.; GALLO, J. R. Métodos de análise química de plantas. Campinas: Instituto Agronômico, 1983. 48 p. (IAC. Boletim Técnico, 78).

BORGES, A. L.; OLIVEIRA, A. M. G.; SOUZA, L. da S. Solos, nutrição e adubação da bananeira. Cruz das Almas: EMBRAPA-CNPMF, 1995. 44 p. (Circular Técnica, 22).

FONTES, P. S. F.; CARVALHO, A. J. C. de; CEREJA, B. S.; MARINHO, C. S.; MONNERAT, P. H. Avaliação do estado nutricional e do desenvolvimento da bananeira Prata-Anâ (Musa spp.) em função da adubação nitrogenada. Revista Brasileira de Fruticultura, Jaboticabal, v. 25, n. 1, p. 156-159, 2003.

INSTITUTO BRASILEIRO DE GEOGRAFIA E ESTATÍSTICA. Produçãa agrícola municipal. 2002. Disponível em: 'Khttp://Www.ibge.gov.bry' Acesso em: $11 \mathrm{dez} .2003$.
INTERNATIONAL FERTILIZER INDUSTRY ASSOCIATION. World fertilizer use manual. Paris, 1992. $404 \mathrm{p}$.

LAHAV, E.; TURNER, D. Banana nutrition. Bern: International Potash Institute, 1983. 62 p. (IPI-Bulletin, 7).

MALAVOLTA, E.; VITTI, G. C.; OLIVEIRA, S. A. de. Avaliação do estado nutricional das plantas: princípios e aplicações. Piracicaba: Associação Brasileira para Pesquisa da Potassa e do Fosfato, 1989. 201 p.

MUNSON, R. D.; NELSON, W. L. Principles and practices in plant analysis. In: WALSH, L. M.; BEATON, J. D. (Eds.). Soil testing and plant analysis. Madison: Soil Science Society of America, 1973. p. 223-248.

SILVA, E. B.; RODRIGUES, M. G. V. Levantamento nutricional dos bananais da região norte de Minas Gerais pela análise foliar. Revista Brasileira de Fruticultura, Jaboticabal, v. 23, n. 3, p. 695-698, 2001.

SILVA, J. T. A.; BORGES, A. L.; DIAS, M. S. C.; COSTA, E. L. da; PRUDÊNCIO, J. M. Diagnóstico nutricional da bananeira 'Prata-Anã' para o Norte de Minas Gerais. Belo Horizonte: EPAMIG, 2002. 16 p. (Boletim Técnico, 70)

TEIXEIRA, L. A. J.; SANTOS, W. R. dos; BATAGLIA, O. C. Diagnose nutricional para nitrogênio e potássio em bananeira por meio do sistema integrado de diagnose e recomendação (DRIS) e de níveis críticos. Revista Brasileira de Fruticultura, Jaboticabal, v. 24, n. 2, p. 530-535, 2002 